\title{
Temperature induced dynamics of water confined between graphene and $\mathrm{MoS}_{2}$
}

Cite as: J. Chem. Phys. 154, 134705 (2021); https://doi.org/10.1063/5.0044123

Submitted: 14 January 2021 • Accepted: 24 March 2021 • Published Online: 05 April 2021

(iD) P. Bampoulis

COLLECTIONS

Paper published as part of the special topic on Fluids in Nanopores
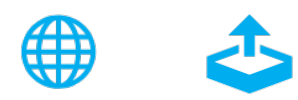

View Online

Export Citation

\section{ARTICLES YOU MAY BE INTERESTED IN}

Molecular fluid flow in $\mathrm{MoS}_{2}$ nanoporous membranes and hydrodynamics interactions The Journal of Chemical Physics 154, 134506 (2021); https://doi.org/10.1063/5.0039963

Structure of water confined between two parallel graphene plates

The Journal of Chemical Physics 150, 124703 (2019); https://doi.org/10.1063/1.5080788

Effects of interfaces on structure and dynamics of water droplets on a graphene surface: A molecular dynamics study

The Journal of Chemical Physics 154, 164704 (2021); https://doi.org/10.1063/5.0046817

Challenge us.

What are your needs for periodic signal detection?

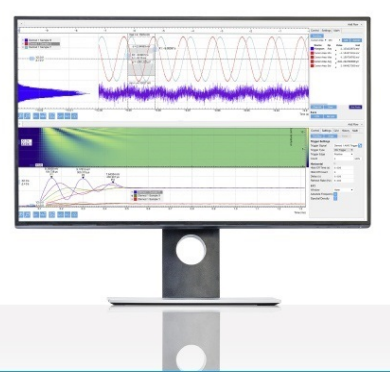

Zurich

- Instruments 


\title{
Temperature induced dynamics of water confined between graphene and $\mathrm{MoS}_{2}$
}

\author{
Cite as: J. Chem. Phys. 154, 134705 (2021); doi: 10.1063/5.0044123 \\ Submitted: 14 January 2021 - Accepted: 24 March 2021 • \\ Published Online: 5 April 2021
}

\section{P. Bampoulis}

\begin{abstract}
AFFILIATIONS
Physics of Interfaces and Nanomaterials, MESA+ Institute for Nanotechnology, University of Twente, P.0. Box 217, 7500AE Enschede, The Netherlands and II. Physikalisches Institut, Universität zu Köln, Cologne D-50937, Germany
\end{abstract}

Note: This paper is part of the JCP Special Topic on Fluids in Nanopores.

a) Author to whom correspondence should be addressed: p.bampoulis@gmail.com

\begin{abstract}
Water trapped between $\mathrm{MoS}_{2}$ and graphene assumes a form of ice composed of two planar hexagonal layers with a non-tetrahedral geometry. Additional water does not wet these ice layers but forms three-dimensional droplets. Here, we have investigated the temperature induced dewetting dynamics of the confined ice and water droplets. The ice crystals gradually decrease in size with increasing substrate temperature and completely vanish at about $80^{\circ} \mathrm{C}$. Further heating to $100^{\circ} \mathrm{C}$ induces changes in water droplet density, size, and shape through droplet coalescence and dissolution. However, even prolonged annealing at $100^{\circ} \mathrm{C}$ does not completely dry the interface. The dewetting dynamics are controlled by the graphene cover. Thicker graphene flakes allow faster water diffusion as a consequence of the reduction of graphene's conformity along the ice crystal's edges, which leaves enough space for water molecules to diffuse along the ice edges and evaporate to the environment through defects in the graphene cover.
\end{abstract}

Published under license by AIP Publishing. https://doi.org/10.1063/5.0044123

\section{INTRODUCTION}

Confined water is relevant in a number of applications in environmental sciences, nanofluidics, biology, lubrication, and sensors. $^{1-4}$ Water diffusion inside nanopores has been explored as a novel drug delivery method. ${ }^{4}$ Intrusion or extrusion of water in or out of nanopores can be used for storage and dissipation of energy. In the case of two-dimensional (2D) materials and nanoelectronics, water trapped at the interfaces of heterostructures influences the electronic properties of the $2 \mathrm{D}$ material, e.g., by doping, affecting device homogeneity and functionality. ${ }^{6-9}$ Investigation of confined water is also interesting from a fundamental point of view as new phases and molecular dynamics can be observed.

Several different types of water and ice structures have been observed and characterized experimentally and theoretically inside different types of confined geometries such as in nanotubes ${ }^{1,18,19}$ and graphene (or other 2D materials) covered water on various types of substrates, e.g., mica, graphene, $\mathrm{Au}$, and $\mathrm{MoS}_{2} \cdot{ }^{10,11,17,20-23}$ The use of a $2 \mathrm{D}$ material as a cover does not only trap water on the substrate of choice but also its conformity allows the use of surface science techniques to experimentally probe this water. ${ }^{20}$
The physicochemical properties of the confined water are much different from its bulk counterparts and depend strongly on several parameters including the hydrophobicity of the confining walls, the applied pressure, the temperature, and the structural characteristics of the surface in direct contact. ${ }^{24-32}$ Properties such as nontetrahedral bonding geometries, anomalous self-diffusion, and complex phase behavior and transitions are only a few of the remarkable characteristics observed in nanoconfined water. ${ }^{24-26}$ Inside hydrophobic confinement, water displays many phases that depend on the exact confinement characteristics ${ }^{1,33}$ as well as fast dynamics. ${ }^{34}$ For example, the large pressure and hydrophobicity of graphene induce a square lattice arrangement for water layers trapped between two graphene sheets. ${ }^{11,35}$ Another form of ice was reported to form at the $\mathrm{MoS}_{2} /$ graphene interface. ${ }^{14}$ This type of ice does not follow the symmetry of the bilayer $\mathrm{I}_{h}$-ice but rather consists of two flat water layers in AA registry with respect to each other. $^{29}$

While significant progress has been made to understand the influence of $2 \mathrm{D}$ material confinement on water and also the influence of water on the 2D material, there are many unexplored aspects mainly because of the rich structure and dynamics of confined water. 
In this article, we explore the adsorption sites of water on $\mathrm{MoS}_{2}$. When these water layers are covered by graphene, they assume a bilayer crystalline ice structure much different from that of conventional $\mathrm{I}_{h}$-ice. Here, we have scrutinized the stability of these ice layers to high temperatures, the induced dynamics of water molecules, and the role of the graphene cover in defining these processes.

\section{METHODS}

Few-layer (FL) graphene flakes were obtained by exfoliation of highly oriented pyrolytic graphite (HOPG ZYA grade, MikroMasch). The graphene flakes were then deposited on top of a freshly cleaved synthesized $2 \mathrm{H}-\mathrm{MoS}_{2}$ (2D semiconductors) substrate by gently pressing them on top of it. Graphene and $\mathrm{MoS}_{2}$ are considered slightly hydrophobic, with reported contact angles, for water droplets in the range of $70^{\circ}-90^{\circ} .^{36}$ The graphene flakes were first located using optical microscopy (DM2500H materials microscope, Leica, Germany) and then characterized with atomic force microscopy (AFM) (Agilent 5100 atomic force microscope, Agilent). The number of graphene layers was determined using the procedure described in detail in Ref. 14, where a fold on the graphene flake is used as a reference to extract the graphene thickness. AFM imaging was done in the intermittent contact amplitude modulated mode (tapping mode) using NANOSENSORS SSSFMR-10 with a nominal spring-constant of $2.8 \mathrm{~N} / \mathrm{m}$ and resonance frequency of $75 \mathrm{kHz}$ inside an environmental chamber. Scanning tunneling microscopy (STM) was done in room temperature and in a relative humidity $(\mathrm{RH})$ controlled environment with an UHV variable temperature AFM/STM (BeetleTM, RHK Technology). The $\mathrm{RH}$ inside the glove box, the AFM environmental chamber, and the STM chamber was controlled using a $N_{2}$ flow with adjustable rates, which was either bubbled through a water bottle or directly purged into the environmental chamber. The RH was measured using a humidity sensor (SENSIRION EK-H4 SHTXX, Humidity Sensors, Eval Kit, SENSIRION, Switzerland), with an accuracy of $1.8 \%$ between $10 \%$ and $90 \% \mathrm{RH}$. The experiments were done at room temperature.

\section{RESULTS AND DISCUSSION}

An AFM image of the freshly cleaved $\mathrm{MoS}_{2}$ surface after exposure to high relative humidity (RH) for 5 min is shown in Fig. 1(a). Higher contrast layers are found across the whole surface and are easily perturbed by the AFM tip. The layers are slightly brighter than the rest of the surface and show a strong contrast in phase imaging compared to the clean $\mathrm{MoS}_{2}$ surface [see the inset of Fig. 1(a)]. This indicates that the tip interacts with a different and softer material than $\mathrm{MoS}_{2}$. The layers are the result of the exposure to high relative humidity as they do not appear in dry surfaces and their coverage seems to scale with the exposure time to high $\mathrm{RH}$, revealing that they are hydration layers. ${ }^{14}$ As shown in Ref. 14, water on $\mathrm{MoS}_{2}$ preferentially adsorbs on surface defects such as step edges and stacking faults ${ }^{37}$ and thus takes an elongated appearance. Here, we identified one more adsorption site, which is of domain boundaries. High-resolution scanning tunneling microscopy imaging of $\mathrm{MoS}_{2}$ before and after exposure to high $\mathrm{RH}$ conditions (5 min at $90 \% \mathrm{RH}$ ) demonstrates water adsorption at domain boundaries [see Figs. 1(b) and $1(\mathrm{c})]$. The water layers expand laterally from these locations and cover the rest of the surface.
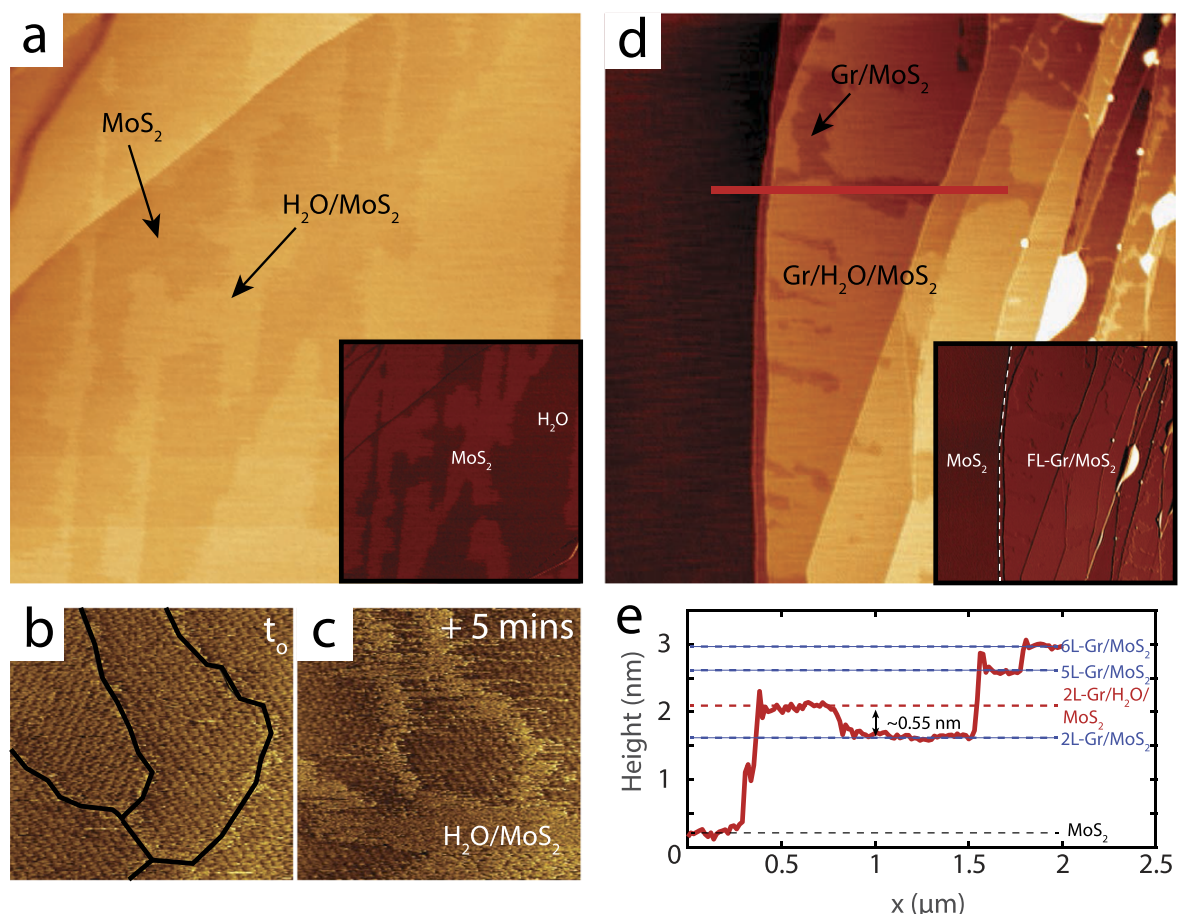

FIG. 1. (a) AFM image $\left(5 \times 5 \mu \mathrm{m}^{2}\right.$, height scale: $1 \mathrm{~nm}$ ) of water layers on the $\mathrm{MoS}_{2}$ surface formed after exposure to high $\mathrm{RH}(>80 \%)$ for $5 \mathrm{~min}$. The inset $\left(5 \times 5 \mu \mathrm{m}^{2}\right)$ is the corresponding phase image showing strong phase contrast between regions with and without water. (b) High-resolution STM image (8 $\times 8 \mathrm{~nm}^{2}$ ) of a region on $\mathrm{MoS}_{2}$ with several domain boundaries. At $t_{0}$, the STM chamber was exposed to $85 \%$ RH. (c) The same region as in (b) after 5 min exposure to high $\mathrm{RH}, 85 \%$. Water layers form and expand from the domain boundaries. (d) AFM image $\left(4 \times 4 \mu \mathrm{m}^{2}\right.$, height: $5 \mathrm{~nm}$ ) of a FL-graphene (Gr) flake on $\mathrm{MoS}_{2}$. Two contrasts can be seen within the same graphene layer thickness, and the brighter regions correspond to intercalated water layers while the darker regions to water free regions. Inset: the corresponding phase image. (e) A line profile taken along the red line in (d) used for the estimation of the thickness of the intercalated water islands. 
Pressing a mechanically exfoliated graphene flake on $\mathrm{MoS}_{2}$ leads to the entrapment of these layers between the two materials. Figure 1(d) shows a few-layer (FL) graphene flake deposited on $\mathrm{MoS}_{2}$ under high humidity conditions $(>80 \% \mathrm{RH})$. In agreement with Ref. 14, water layers and droplets are found across the graphene/ $\mathrm{MoS}_{2}$ interface. Phase imaging [inset of Fig. 1(d)] reveals no contrast between regions with and without water layers, indicating that the tip interacts always with the same surface of graphene and that these water layers are indeed under the graphene cover. In comparison, the bare $\mathrm{MoS}_{2}$ regions have a phase contrast compared to the graphene/ $\mathrm{MoS}_{2}$ regions. The water layers have a height of about $0.55 \mathrm{~nm}$ [see the line profile in Fig. 1(e)]. As described in Ref. 14, these water islands can be explained assuming a crystalline phase that consists of two hexagonal planar layers in the AA registry with respect to each other and with a non-tetrahedral geometry, similar to the two-layer ice on graphene on $\operatorname{Pt}(111)$, hereby referred as two-layer (2L) ice. In this structure, every water molecule forms three in-plane hydrogen bonds with neighboring water molecules and one out-of-plane hygrogen bond with the opposite water layer. The $2 \mathrm{~L}$ ice has a self-closed hydrogen bonding network with low surface energy due to the maximized number of hydrogen bonds. ${ }^{29,38,39}$ The lack of free dangling/hydrogen bonds makes this ice structure more hydrophobic, which explains the lack of a third layer and the preference toward formation of 3D droplets [see Fig. 2(a)].

Understanding the physics governing water diffusion at the interface between graphene and $\mathrm{MoS}_{2}$ will provide new insights into water diffusion in hydrophobic confinements and the dynamics of the $2 \mathrm{~L}$ ice. We have investigated the influence of humidity and temperature on the structure and dynamics of the $2 \mathrm{~L}$ confined ice. Lowering the RH in the sample's environment to below $4 \%$ (using a dedicated environmental chamber) did not induce any measurable changes in the shape and size of both the ice layers and the water droplets. The water structures remained unaltered during overnight low $\mathrm{RH}$ exposure. This is in contrast to water confined between graphene and mica or other hydrophobichydrophilic confinements in which water responds fast to changes in the environmental $\mathrm{RH} .{ }^{10,40-42} \mathrm{We}$ attribute this finding to the hydrophobic character of the interface that significantly hinders water diffusion in and out of the interface without a substantial driving force. In this case, the pressure gradient is not sufficient to induce de-intercalation of water. We note that water intercalation
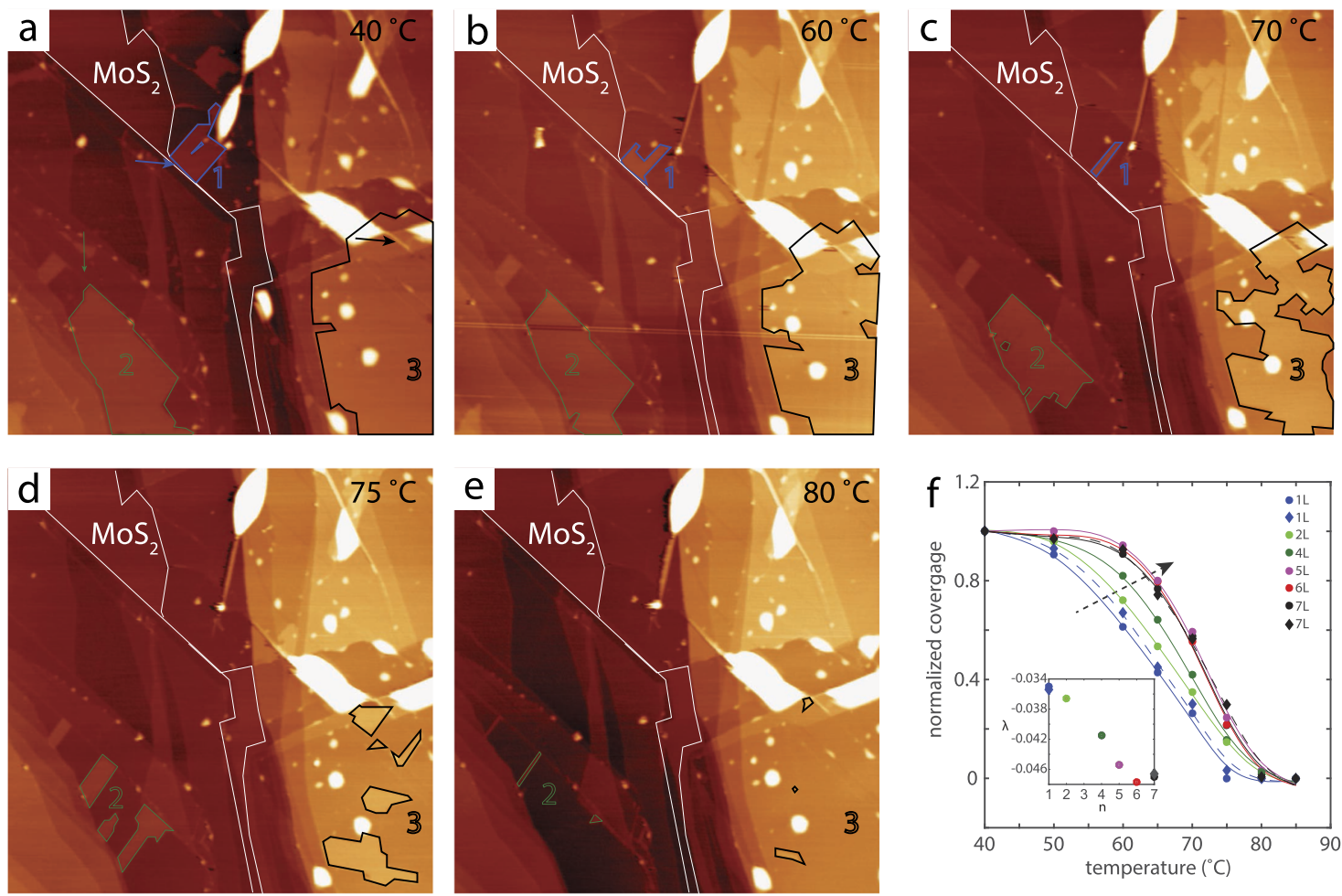

FIG. 2. (a) AFM images $\left(5.5 \times 5.5 \mu \mathrm{m}^{2}\right.$, height scale: $10 \mathrm{~nm}$, the big droplets are clipped) of a graphene flake with various thicknesses on $\mathrm{MoS}_{2}$. $2 \mathrm{~L}$ ice islands are marked with the solid lines and are under the graphene flake. The blue island is under $1 \mathrm{~L}$ graphene $(\mathrm{Gr})$, the green under $4 \mathrm{~L}$ graphene, and the black under $7 \mathrm{~L} \mathrm{Gr}$. The arrows indicate possible defects (step edge with blue and wrinkle with green and black) where water can communicate with the environment. Moreover, various sizes of water droplets are also on top and at the edges of the $2 \mathrm{~L}$ ice. (b)-(e) Temperature evolution of the confined water structures, (b) the surface at $60^{\circ} \mathrm{C},(\mathrm{c})$ at $70^{\circ} \mathrm{C},(\mathrm{d})$ at $75^{\circ} \mathrm{C}$, and $(\mathrm{e})$ at $80^{\circ} \mathrm{C}$. (f) The normalized island coverage as a function of the substrate temperature for ice crystals under $1 \mathrm{~L}, 2 \mathrm{~L}, 4 \mathrm{~L}, 5 \mathrm{~L}, 6 \mathrm{~L}$, and $7 \mathrm{~L}$ of graphene. The inset shows the slope of the coverage vs the temperature graph as a function of the number of graphene layers. The experimental data marked with filled circles represent ice islands confined in the same graphene/MoS 2 sample while with a diamond marker represent data acquired from a different but similarly prepared sample. 
when the dry graphene/ $\mathrm{MoS}_{2}$ interface (sample prepared under $<\% \mathrm{RH})$ is exposed to high $\mathrm{RH}(80 \%-90 \%)$ for $8 \mathrm{~h}$ was also not possible.

In order to assist water de-intercalation, we exposed the graphene/ $\mathrm{MoS}_{2}$ sample to a gradual increase of temperature, up to $100^{\circ} \mathrm{C}$. The heating rate was set to $1^{\circ} \mathrm{C} / \mathrm{min}$. Every $5^{\circ} \mathrm{C}$, an AFM image was captured. For this to be possible, the tip and sample need to reach the thermal equilibrium, and thus, the sample was maintained at a fixed temperature for $15 \mathrm{~min}$ before imaging. As a reference, we have marked the contour of three $2 \mathrm{~L}$ ice islands under 1L graphene (blue contour, island "1"), $4 \mathrm{~L}$ graphene (green contour, island " 2 "), and $7 \mathrm{~L}$ graphene (black contour, island " 3 "). Heating the sample from room temperature up to $50^{\circ} \mathrm{C}$ has no measurable effect on the $2 \mathrm{~L}$ ice. After $50^{\circ} \mathrm{C}$, the ice starts to shrink. As seen in panels (c)-(e) of Fig. 2, further increase of the temperature leads to a faster decrease of the islands' size. Heating to $80^{\circ} \mathrm{C}$ completely removes most of the islands with only a few remaining residual layers that also disappear in the next heating step to $85^{\circ} \mathrm{C}$.

The $2 \mathrm{~L}$ ice crystals start to de-intercalate at $\sim 50^{\circ} \mathrm{C}$, setting the mark for water detachment from the $2 \mathrm{~L}$ ice, diffusion of water molecules along its edges, and evaporation to the environment. Water evaporation appears to happen through defects in the graphene cover. We have identified several types of defects that could provide such exits and direct communication to the environment. For example, the water molecules from the ice island "1" escape from the graphene edge (marked with a blue arrow). Water molecules from island "2" escape from a graphene wrinkle stretching across the whole $4 \mathrm{~L}$ graphene flake (marked with a green arrow). The 2L ice " 3 " (underneath 7L graphene) appears to have its main escape path through the wrinkles formed by the two large droplets (black arrow). Therefore, and similar to other studies on confined water between $2 \mathrm{D}$ materials, ${ }^{17}$ water communicates with the environment through defects in the upper $2 \mathrm{D}$ material cover, in this case, the graphene.

To quantitatively analyze the influence of temperature on the dynamics of the $2 \mathrm{~L}$ ice, we plotted the normalized ice coverage, $\theta$, defined as $\theta=\alpha_{T} / \alpha_{i}$, where $\alpha_{T}$ is the area occupied by the ice layer at each temperature step and $\alpha_{i}$ is the ice area at RT, as a function of temperature [Fig. 2(f)]. For graphene flakes with thickness between one and seven layers, water diffusion and evaporation set in between 50 and $60^{\circ} \mathrm{C}$ and end at $\sim 80^{\circ} \mathrm{C}$, where the $2 \mathrm{~L}$ ice completely disappears. However, the slope of the de-intercalation shows a dependency on the graphene cover thickness. This is also demonstrated in the inset of Fig. 2(f) where we plotted the slope of each curve as a function of the number of graphene layers (n). While water deintercalation starts to really take over at slightly higher temperatures for thicker flakes (possibly due to the better thermal insulation by the thicker graphene), the slope of the curve is much steeper. The same trend has also been measured in other samples, which are indicated with diamond markers in Fig. 2(f). The water de-intercalates at a faster rate under thicker graphene flakes.

The small thickness of the graphene cover allows it to conform the trapped $2 \mathrm{~L}$ ice and water droplets and to adapt to the surface corrugation with great detail, which enables visualization of these trapped water structures with scanning probe techniques. However, this conformity leaves little space to the edge water molecules of the trapped 2L ice to move, and the water molecules must overcome the resistance imposed by the graphene. The radial retraction [see
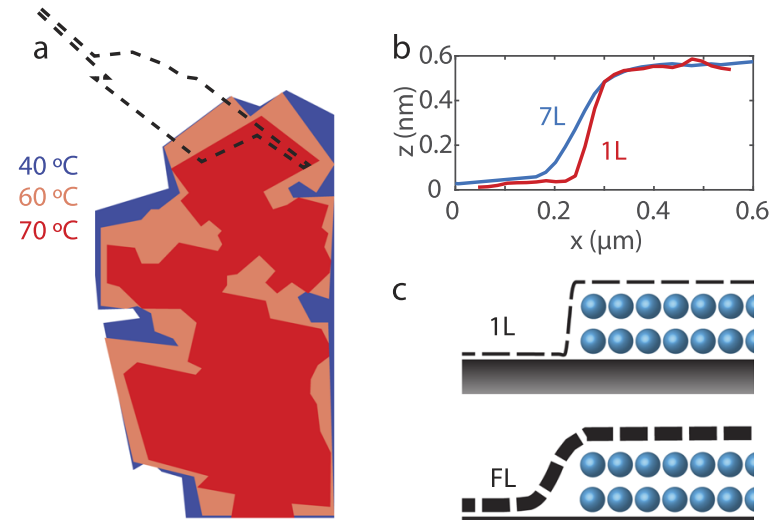

C

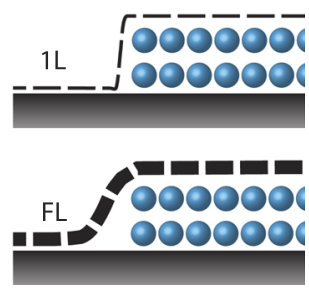

FIG. 3. The contour of the ice crystal "3" under $7 \mathrm{~L}$ graphene shown in Fig. 2 for temperatures of 40,60 , and $70^{\circ} \mathrm{C}$ (b) Line profiles taken across the edges of confined ice located below $1 \mathrm{~L}$ and $7 \mathrm{~L}$ of graphene, respectively. The difference in the profiles is explained by the non-perfect conformity of the ice boundaries by the thicker graphene, which is schematically shown in the cartoon of (c). The top panel of (c) shows the more accurate conformity of the ice edges by a $1 \mathrm{~L}$ graphene, while at the bottom panel, a gap forms between the ice edges and the thicker graphene cover. The blue spheres represent water molecules.

Fig. 3(a)] of the ice layers suggests that water diffusion along the ice edges is indeed a relevant process. The water molecules at the edges of the crystal are the first to detach, diffuse along its edges until they find an appropriate defect, and evaporate to the environment. Water diffusion along the edges of the ice and toward the escape paths to the environment under $1 \mathrm{~L}$ graphene is expected to be slow. This is because 1L graphene follows the ice crystal with great detail due to its high conformity, which leaves little space for water molecules after their initial detachment to diffuse along the ice edges, before reaching a defect, and escape to the environment. Increasing the thickness of graphene decreases its bending ability and conformity, increasing the gap at the interface of regions with and without a $2 \mathrm{~L}$ ice, and this gap gets larger with increasing graphene thickness. ${ }^{13}$ This is shown in the line profiles of Fig. 3(b) taken across the edge of an ice crystal below 1L and 7L of graphene and the schematic of Fig. 3(c). The water molecules at the edges of the $2 \mathrm{~L}$ ice below thicker graphene have more space to diffuse along the edges of the ice, and thus, the ice melts at a faster rate underneath thicker flakes. For thicker than $5 \mathrm{~L}$ of graphene, even though the conformity should decrease further, the slope of the coverage vs temperature plot increases with a much slower rate. At this point, the graphene-ice interface gap is large enough for water diffusion that the additional opening has little to no effect in the observed dynamics.

The dynamics of the confined water structures underline the effect of the 2D material cover in defining the de-wetting processes. Dewetting occurs through pores and defects in the graphene layer such as wrinkles and step edges. Water mobility is slower for thinner graphene flakes due to the better conformity of the ice edges by the thinner graphene flakes.

The ice layers have already been melted and evaporated away around $80^{\circ} \mathrm{C}$. The water droplets seen in Fig. 2 have not shown any measurable differences up to $80^{\circ} \mathrm{C}$. Further heating of the sample 
to $100^{\circ} \mathrm{C}$ induces some changes. Figures $4(\mathrm{a})-4(\mathrm{e})$ show continuation of the stepwise heating of the sample with steps of $5^{\circ} \mathrm{C}$, from $80^{\circ} \mathrm{C}$ up to $100^{\circ} \mathrm{C}$. We have marked several events induced by heating, which can be categorized to (i) droplet coalescence and (ii) droplet shrinkage/dissolution. Assuming that enough energy is provided (e.g., by heating), the water droplets have some freedom to coalesce or even slowly evaporate to the environment. The graphene layer on top even though protects the water droplets from tip perturbations and confines them to stable entities, at the same time when enough force is exerted, its bending ability allows for some adaptation. While the elevated temperature is enough to induce changes in the water droplets, this mild annealing does not completely remove the intercalated water. In fact, prolonged $(1 \mathrm{~h})$ annealing at $100^{\circ} \mathrm{C}$ [Fig. 4(f)] does not make any difference, and the remaining water droplets are left unchanged. This could have implications in the
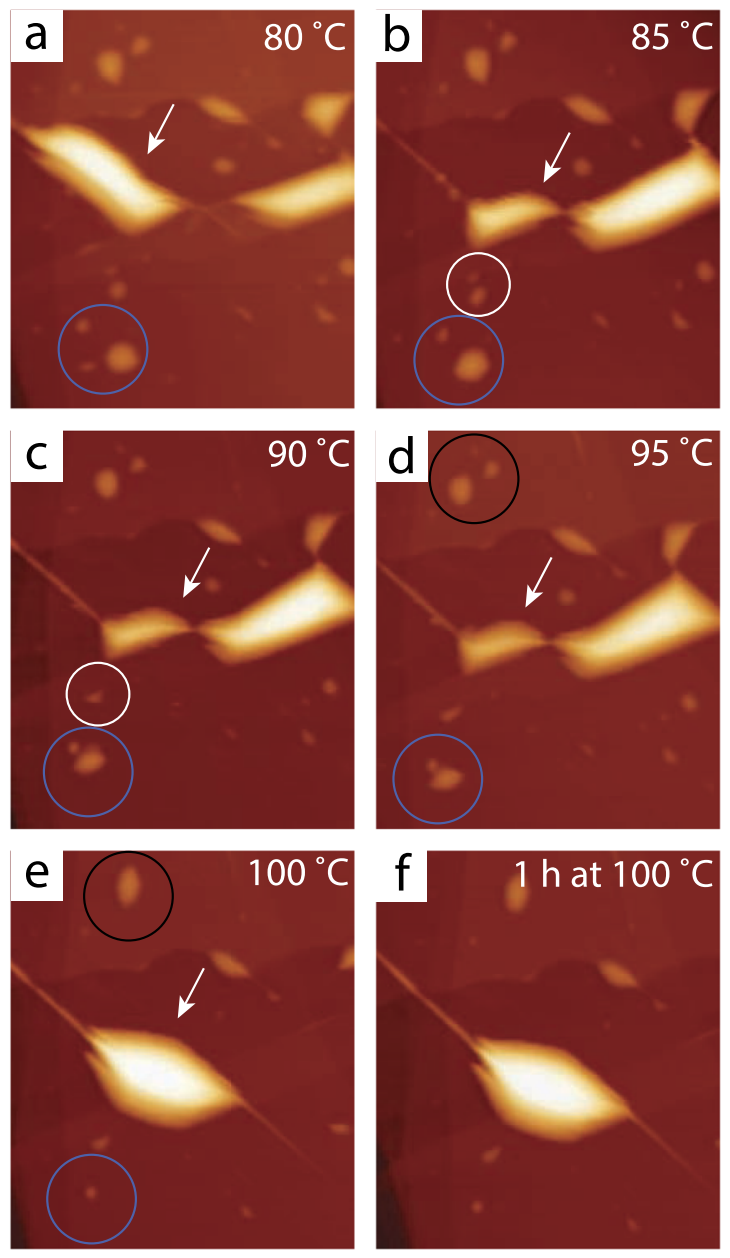

FIG. 4. AFM images $\left(2.5 \times 3 \mu \mathrm{m}^{2}\right.$, height scale: $\left.30 \mathrm{~nm}\right)$ of various size water droplets trapped between a FL-graphene (Gr) flake on $\mathrm{MoS}_{2}$ at sample temperatures (a) $80^{\circ} \mathrm{C}$, (b) $85^{\circ} \mathrm{C}$, (c) $90^{\circ} \mathrm{C}$, (d) $95^{\circ} \mathrm{C}$, (e) $100^{\circ} \mathrm{C}$, and (f) after $1 \mathrm{~h}$ heating at $100^{\circ} \mathrm{C}$. The images show several events of coalescence and droplet shrinkage, marked with circles or indicated by arrows.
2D material community where removal of water residuals formed during transfer processes is important to obtain homogeneous samples.

In conclusion, water layers trapped between graphene and $\mathrm{MoS}_{2}$ preferentially adsorb on surface defects of $\mathrm{MoS}_{2}$, such as step edges and domain boundaries. The evaporation dynamics of the confined water molecules are defined by the graphene cover. Water communicates with the environment through graphene defects such as step edges and wrinkles. The dynamics of the water molecules from the confined $2 \mathrm{~L}$ ice during heating depend on the graphene thickness. Water molecules under thinner graphenes have less freedom to move along the ice edges and reach defects in the graphene cover to evaporate to the environment. This is because thinner graphene flakes have lower bending energy and thus adapt to the surface corrugation with greater detail, but because of this, they leave less space to the edge water molecules of the $2 \mathrm{~L}$ ice to move. The water molecules have to overcome the resistance imposed by the graphene. Water droplets react to heating only after $80^{\circ} \mathrm{C}$. Several coalescence and droplet dissolution events were imaged to occur under graphene. However, even prolonged annealing to $100^{\circ} \mathrm{C}$ is not sufficient to completely remove the trapped water droplets and dewet the interface. Our findings reveal the intriguing dynamics of water between graphene and $\mathrm{MoS}_{2}$.

\section{ACKNOWLEDGMENTS}

P.B. acknowledges financial support from the Alexander von Humboldt Foundation through a Humboldt Research Fellowship and thanks Harold J. W. Zandvliet for fruitful discussions.

\section{DATA AVAILABILITY}

The data that support the findings of this study are available from the corresponding author upon reasonable request.

\section{REFERENCES}

${ }^{1}$ D. Takaiwa, I. Hatano, K. Koga, and H. Tanaka, Proc. Natl. Acad. Sci. U. S. A. 105, 39 (2008).

${ }^{2}$ C. Lee, Q. Li, W. Kalb, X. Z. Liu, H. Berger, R. W. Carpick, and J. Hone, Science 328, 76 (2010).

${ }^{3}$ S. Prakash, A. Piruska, E. N. Gatimu, P. W. Bohn, J. V. Sweedler, and M. A. Shannon, IEEE Sens. J. 8, 441 (2008).

${ }^{4}$ V. V. Chaban and O. V. Prezhdo, ACS Nano 5, 5647 (2011).

${ }^{5}$ V. Eroshenko, R.-C. Regis, M. Soulard, and J. Patarin, J. Am. Chem. Soc. 123, 8129 (2001).

${ }^{6}$ J. Shim, C. H. Lui, T. Y. Ko, Y.-J. Yu, P. Kim, T. F. Heinz, and S. Ryu, Nano Lett. 12, 648 (2012).

${ }^{7}$ Y. Wang and Z. Xu, ACS Appl. Mater. Interfaces 8, 1970 (2016).

${ }^{8}$ H. Lin, J. D. Cojal González, N. Severin, I. M. Sokolov, and J. P. Rabe, ACS Nano 14, 11594 (2020).

${ }^{9}$ Z. Li, S. Li, H.-Y. T. Chen, N. Gao, K. Schouteden, X. Qiang, J. Zhao, S. Brems, C. Huyghebaert, and C. Van Haesendonck, J. Phys. Chem. Lett. 10, 3998 (2019).

${ }^{10}$ N. Severin, P. Lange, I. M. Sokolov, and J. P. Rabe, Nano Lett. 12, 774 (2012).

${ }^{11}$ G. Algara-Siller, O. Lehtinen, F. C. Wang, R. R. Nair, U. Kaiser, H. A. Wu, A. K. Geim, and I. V. Grigorieva, Nature 519, 443 (2015).

${ }^{12}$ P. Bampoulis, M. H. Siekman, E. S. Kooij, D. Lohse, H. J. W. Zandvliet, and B. Poelsema, J. Chem. Phys. 143, 034702 (2015). 
${ }^{13}$ P. Bampoulis, D. Lohse, H. J. W. Zandvliet, and B. Poelsema, Appl. Phys. Lett. 108, 011601 (2016).

${ }^{14}$ P. Bampoulis, V. J. Teernstra, D. Lohse, H. J. W. Zandvliet, and B. Poelsema, J. Phys. Chem. C 120, 27079 (2016).

${ }^{15}$ E. Dollekamp, P. Bampoulis, B. Poelsema, H. J. W. Zandvliet, and E. S. Kooij, Langmuir 32, 6582 (2016).

${ }^{16}$ K. Sotthewes, P. Bampoulis, H. J. W. Zandvliet, D. Lohse, and B. Poelsema, ACS Nano 11, 12723 (2017).

${ }^{17}$ P. Bampoulis, K. Sotthewes, E. Dollekamp, and B. Poelsema, Surf. Sci. Rep. 73, 233 (2018).

${ }^{18}$ A. Kalra, S. Garde, and G. Hummer, Proc. Natl. Acad. Sci. U. S. A. 100, 10175 (2003).

${ }^{19}$ M. H. Köhler and C. Gavazzoni, J. Phys. Chem. C 123, 13968 (2019).

${ }^{20} \mathrm{~K}$. Xu, P. Cao, and J. R. Heath, Science 329, 1188 (2010).

${ }^{21}$ P. Cao, J. O. Varghese, K. Xu, and J. R. Heath, Nano Lett. 12, 1459 (2012).

${ }^{22}$ D. A. Sanchez, Z. Dai, P. Wang, A. Cantu-Chavez, C. J. Brennan, R. Huang, and N. Lu, Proc. Natl. Acad. Sci. U. S. A. 115, 7884 (2018).

${ }^{23}$ X. Cai, W. J. Xie, Y. Yang, Z. Long, J. Zhang, Z. Qiao, L. Yang, and Y. Q. Gao, J. Chem. Phys. 150, 124703 (2019).

${ }^{24}$ S. Han, M. Y. Choi, P. Kumar, and H. E. Stanley, Nat. Phys. 6, 685 (2010).

${ }^{25}$ S. Chakraborty, H. Kumar, C. Dasgupta, and P. K. Maiti, Acc. Chem. Res. 50, 2139 (2017).

${ }^{26}$ H. Yoshida, V. Kaiser, B. Rotenberg, and L. Bocquet, Nat. Commun. 9, 1496 (2018).

${ }^{27}$ A. Verdaguer, G. M. Sacha, H. Bluhm, and M. Salmeron, Chem. Rev. 106, 1478 (2006).

${ }^{28}$ P. J. Feibelman, Phys. Today 63(2), 34 (2010).
${ }^{29}$ G. A. Kimmel, J. Matthiesen, M. Baer, C. J. Mundy, N. G. Petrik, R. S. Smith, Z. Dohnálek, and B. D. Kay, J. Am. Chem. Soc. 131, 12838 (2009).

${ }^{30}$ S. Ruiz-Barragan, D. Muñoz-Santiburcio, and D. Marx, J. Phys. Chem. Lett. 10, 329 (2018).

${ }^{31}$ N. Si, T. Shen, D. Zhou, Q. Tang, Y. Jiang, Q. Ji, H. Huang, W. Liu, S. Li, and T. Niu, ACS Nano 13, 10622 (2019).

${ }^{32}$ B. Cao, E. Xu, and T. Li, ACS Nano 13, 4712 (2019).

${ }^{33}$ N. Naguib, H. Ye, Y. Gogotsi, A. G. Yazicioglu, C. M. Megaridis, and M. Yoshimura, Nano Lett. 4, 2237 (2004).

${ }^{34}$ T. A. Pascal, W. A. Goddard, and Y. Jung, Proc. Natl. Acad. Sci. U. S. A. 108, 11794 (2011)

${ }^{35}$ W. Zhu, Y. Zhu, L. Wang, Q. Zhu, W.-H. Zhao, C. Zhu, J. Bai, J. Yang, L.-F. Yuan, H. Wu et al., J. Phys. Chem. C 122, 6704 (2018).

${ }^{36}$ A. P. S. Gaur, S. Sahoo, M. Ahmadi, S. P. Dash, M. J.-F. Guinel, and R. S. Katiyar, Nano Lett. 14, 4314 (2014).

${ }^{37}$ P. Bampoulis, K. Sotthewes, M. H. Siekman, and H. J. W. Zandvliet, ACS Appl. Mater. Interfaces 10, 13218 (2018).

${ }^{38}$ D. Stacchiola, J. B. Park, P. Liu, S. Ma, F. Yang, D. E. Starr, E. Muller, P. Sutter, and J. Hrbek, J. Phys. Chem. C 113, 15102 (2009).

${ }^{39}$ K. Koga, X. C. Zeng, and H. Tanaka, Phys. Rev. Lett. 79, 5262 (1997).

${ }^{40}$ J. Song, Q. Li, X. Wang, J. Li, S. Zhang, J. Kjems, F. Besenbacher, and M. Dong, Nat. Commun. 5, 4837 (2013).

${ }^{41}$ J. S. Kim, J. S. Choi, M. J. Lee, B. H. Park, D. Bukhvalov, Y. W. Son, D. Yoon, H. Cheong, J. N. Yun, Y. Jung, J. Y. Park, and M. Salmeron, Sci. Rep. 3, 2309 (2013).

${ }^{42}$ O. Ochedowski, B. K. Bussmann, and M. Schleberger, Sci. Rep. 4, 6003 (2014). 